\title{
Organogene Serie einer pleistozänen Warmzeit vom Typ Bederkesa
}

\author{
Von R. HalliK, Hamburg \\ (Mitteilung aus dem Geologischen Landesamt Hamburg Nr. 66)
}

Mit 3 Abbildungen

$\mathrm{Z}$ us a m me n f a s ung. Eine organogene Serie im Geeste-Elbe-Kanal bei Bederkesa (Kreis Wesermünde) wird moorstratigraphisch und pollenanalytisch untersucht. Zum ersten Mal wurden dabei neben Ubereinstimmungen mit organogenen Bildungen des Eems gravierende Abweichungen festgestellt. Zwischen zweien sich ergebenden Deutungsmöglichkeiten bliebe in Anbetracht dieses Befundes zu wählen: entweder eine Erklärung dieser Abweichungen vom "normalen“ Eem-Bild mit lokalen ökologischen Bedingungen, oder die Annahme einer weiteren, bisher unbekannt gebliebenen, quartären Warmzeit. Sowohl die Aussichten auf eine ausreichende ökologische Erklärung als auch die Wahrscheinlichkeit einer hinsichtlich der Vegetationsentwicklung einer der EemZeit in vielem ähnlichen Warmzeit werden behandelt.

$\mathrm{Summary}$. An organogenous series of pleistocene age has been studied both in moorestratigraphical and pollenanalytical sense. Partly wide analogy with the type of Eem-interglacial was found. On the other hand several lines in development of vegetation indicate a climatic change being different from that of the Eem-type known until now. Two alternativ interpretations were discussed:

1. the possibility of local ecologic conditions causing the differencies mentioned above, or

2. this type being a fundamentally new one, hitherto undiscovered in research on pleistocene organogenous layers.

Während der Erweiterungs- und Vertiefungsarbeiten des Geeste-Elbe-Kanals seitens der Neubau-Abteilung Bederkesa der Niedersächsischen Wasserwirtschaftsverwaltung war im Sommer 1959 im alten Kanal eine pleistozäne organogene Schichtserie aufgeschlossen. Der Neubau-Abteilung, besonders Herrn Gross, ist die Kenntnis von diesem Aufschluß zu verdanken. Ebenso großer Dank gebührt den Genannten für weitgehende Unterstützung bei der Entnahme von Proben und die freundliche Bereitstellung eines Bohrprahmes

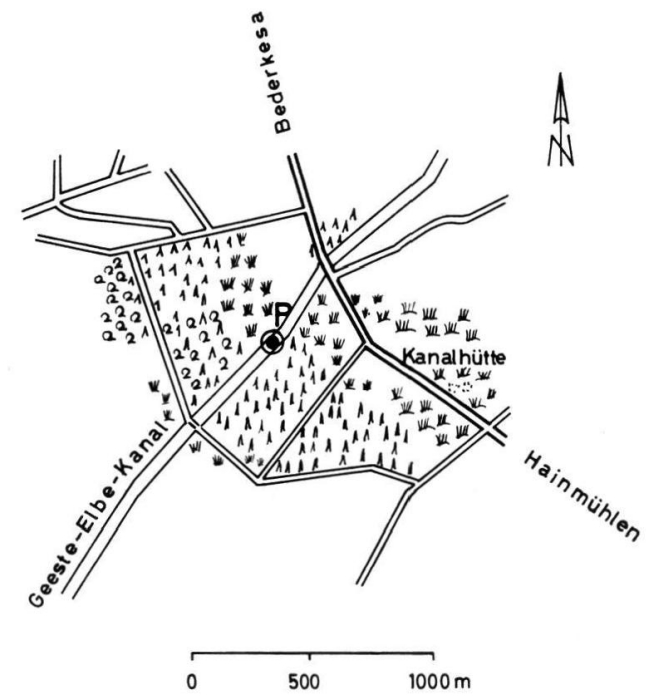

Abb. 1. P = Lagepunkt des untersuchten Vorkommens Bederkesa, R 3490 030, H 5940020. 
im Sommer 1960, mit Hilfe dessen nach Beendigung der Arbeiten von der Sohle des neuen Kanals aus noch weitere Bohrkerne mit Hilfe eines holländischen Marschenlöffels gefördert werden konnten.

Die mineralischen Schichten im Hangenden waren während beider Besuche der Lokalität nicht aufgeschlossen. Ca. $50 \mathrm{~m}$ vom Punkt der Probenentnahme entfernt war in einer Aufgrabung kiesiger Sand sichtbar, der auch an dieser Stelle das Hangende bilden dürfte. Die organogene Schichtserie umfaßt die vollständige Verlandungsfolge eines stehenden Gewässers während einer der pleistozänen Warmzeiten oder Interglaziale. Das aufgeschlossene Profil weist die nachstehende Verlandungsfolge auf:

Zum Nullpunkt wurde die alte Kanalsohle gewählt.

$0-50 \mathrm{~cm}$ Sphagnum-Torf mit Pinus und Picea

$-106 \mathrm{~cm}$ Carex-Torf, im oberen Teil zunehmend Sphagnum, Pinus und Picea

$-145 \mathrm{~cm}$ Alnus- und Betula-Bruchwaldtorf

$-190 \mathrm{~cm}$ Thelypteris-Torf mit Carex

$-215 \mathrm{~cm}$ Grobdetritus-Gyttja.

Die Bohrung von der neuen Kanalsohle aus zeigte weit überwiegend die limnische Serie:

$$
\begin{array}{ll}
0=\text { neue Kanalsohle } \\
0-28 \mathrm{~cm} & \text { Thelypteris-reicher Carex-Torf } \\
-171 \mathrm{~cm} & \text { Grobdetritus-Gyttja } \\
-332 \mathrm{~cm} & \text { Feindetritus-Gyttja } \\
-368 \mathrm{~cm} & \text { Feinsandiger Schluff. }
\end{array}
$$

Das Fehlen einer gesicherten Kontinuität vom Aufschluß zu den Bohrproben veranlaßte die Darstellung des Pollendiagramms in zwei Abbildungen (Abb. 2 und 3). In beiden Fällen ist aber der limnisch-telmatische Kontakt sichtbar, wobei dieser anscheinend nicht synchron ist. Auf einige Besonderheiten dieses Profils wäre hinzuweisen:

1. Der schluffige Feinsand im liegenden Teil des Profils ist als Sediment der Endphase einer voraufgegangenen Kaltzeit aufzufassen.

Die Pollenproduktion einer autochthonen Vegetation ist so gering, daß diese mengenmäßig noch weit vom „sekundären“, hauptsächlich tertiären Pollen überlagert wird. Hier liegt der relativ seltene Fall vor, daß die Bildungen eines stehenden Gewässers bereits seit einer Kaltzeit nachzuweisen sind. Das zunächst gebildete Sediment unterscheidet sich in keiner Beziehung von einer allgemein bekannten glaziären Beckenfüllung. Diese setzt sich nach oben hin unter Zunahme und schließlich im Überwiegen des organischen Anteils in der bereits einer Warmzeit zuzuordnenden Gyttja fort.

2. Die telmatische Verlandungsfolge entspricht dem Typus eines mesotrophen Gewässers, denn über der Gyttja folgt unmittelbar ein Thelypteris-reicher Niedermoortorf. Unter diesen Umständen ist die Annahme einer Schwingrasenbildung durchaus möglich. Die mehrfach gemachte Beobachtung, daß in einem Thelypteris-reichen Torf eine selektive Zerstörung des Pollens zugunsten des Coniferen-Pollens auftritt, ist im vorliegenden Fall nur schwer wieder zu finden. Auffällig ist allerdings der steile Anstieg der Pollenkurve von Picea, der hier (Abb. 2) genau mit dem limno-telmatischen Kontakt zusammenfällt.

3. Bekanntlich erfolgt das weitere Wachstum eines Moores nach Erreichen der Endphase einer Verlandungsfolge in Form des Bruchwaldtorfes in der Regel durch die Veränderung des Klimas in der ausklingenden Warmzeit. Unter den vielfach beobachteten Verhältnissen während der Eem-Warmzeit findet sich im Hangenden des Bruchwaldtorfes stets ein Sphagnum-Torf, der schließlich in eine minerotraphente torfbildende Pflanzengesellschaft der subarktischen Klimaphase übergeht. Im vorliegenden Falle ist die rekurrente Entwicklung noch wesentlich markanter. Und zwar folgt ein 
Bederkesa 1960

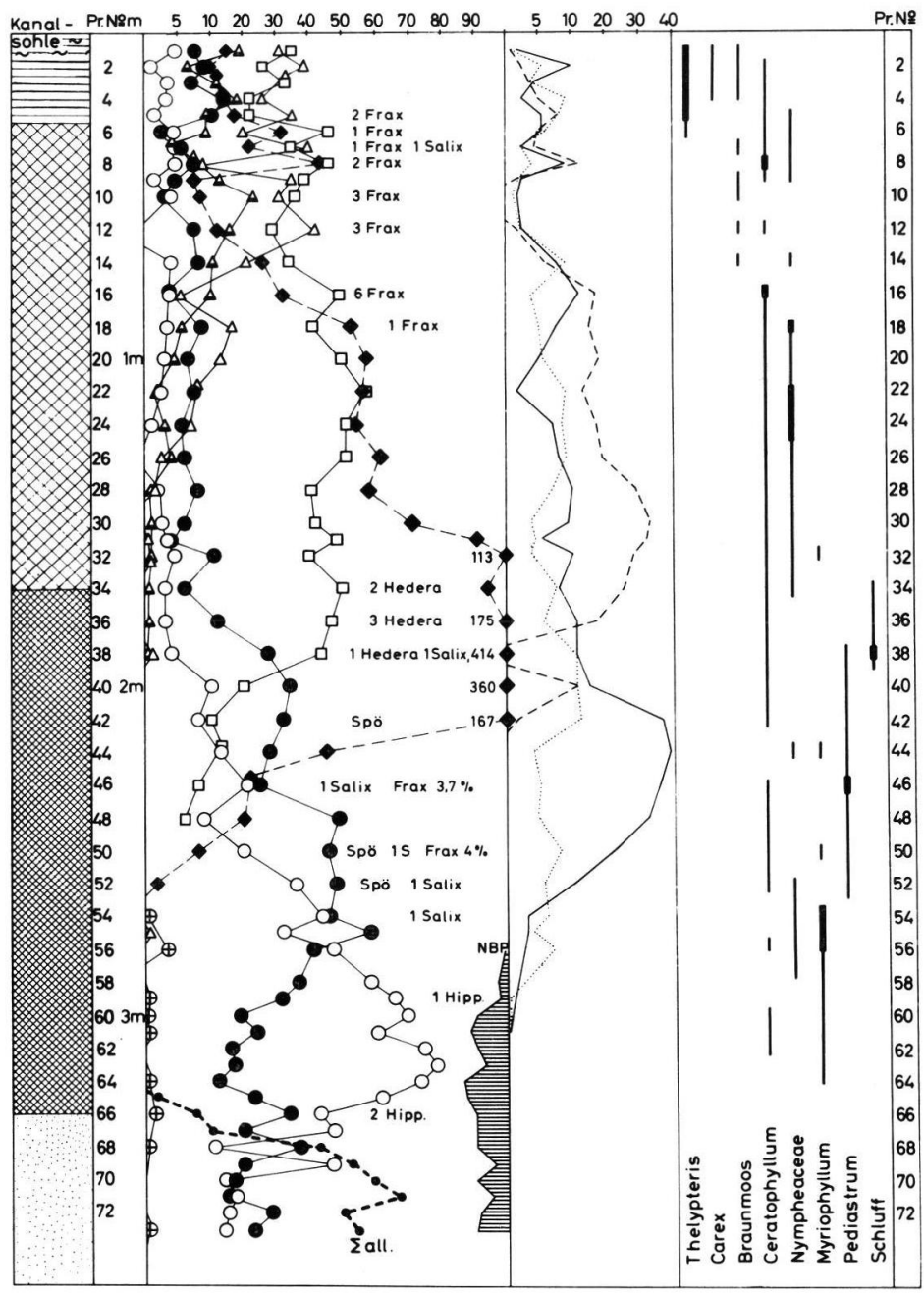

Abb. 2. Pollendiagramm der erbohrten Serie. In beiden Diagrammen wurden die konventionellen Signaturen benutzt. Hipp. = Hippophae, Frax $=$ Fraxinus (Angaben in absoluten Zahlen), all. = Summe des allochthonen (= sekundären) Pollens.

minerotraphenter Carex-Torf, der sehr bald eine merkliche Beimengung von Sphagnum (wahrscheinlich Sectio Subsecunda) aufweist und in der oberen Hälfte ConiferenHolzelemente enthält, demnach mit Pinus und Picea bestanden war. Auch der nachfolgende Sphagnum-Torf läßt einen Coniferen-Bestand erkennen.

Diese sedimentologische (sensu lato) Erörterung ist eine notwendige Voraussetzung für die Interpretation des Pollendiagramms.

Wie bereits ausgeführt, setzt sich die organogene Sedimentation unmittelbar aus einem glaziären Beckenschluff fort (Abb. 2). Das entsprechende Pollenbild ist demzufolge recht unklar. Im Pollendiagramm herrscht der „sekundäre“ Pollen mit 60\% der Gesamtsumme vor. Die Summe des NBP-Pollens ist niedrig. Erst mit dem eigentlichen Be- 
ginn der Warmzeit, die sich auch sedimentologisch abzeichnet, beginnt auch die gut erkennbare Waldentwicklung mit einem Birkenwald. Analog zu den Diagrammen des SpätGlazials muß der Anteil an Pinus-Pollen zunächst auf Ferntransport zurückgeführt werden. Die Beteiligung der NBP mit knapp $10 \%$ der Gesamtsumme ist überraschend niedrig, obgleich ein leichter Anstieg zu verzeichnen ist.

Auffallend ist das Vorkommen. von Pinus-Nadelepidermen mit den typischen Spaltöffnungsapparaten (im Diagramm „Spö“) während der maximalen Vertretung des PinusPollens, die neben den Pollenmaximum eine authochthone Kiefernbewaldung unterstreichen.

Im weiteren Verlauf scheint das Pollendiagramm durchaus dem der hinreichend bekannten Entwicklung des Eem zu entsprechen. Bereits in diesem Abschnitt treten gewisse Abweichungen von einem „normalen“ Eem-Bild auf. Wenn auch die Abhängigkeit eines pollenanalytisch ermittelten Waldbildes von der Fazies berücksichtigt wurde, so muß für die limnische Phase - besonders im Falle fast rein organogener Gyttjen — die geringste Verzerrung angenommen werden. Auffallend sind: die extrem hohe CorylusBeteiligung (die doppelte Menge eines normalen Eem-Bildes), das relativ frühe Auftreten von Tilia (allerdings nur sporadisch), das relativ hohe Tilia-Maximum und schließlich das relativ frühe Auftreten von Carpinus. Letztere ist zwar geschlossen, aber in geringer prozentualer Beteiligung anwesend. Noch wesentlicher ist aber die sehr schnelle PiceaAusbreitung, die Carpinus nicht zur maximalen Entfaltung kommen läßt. Für diese Abweichungen vom „normalen“ Eem-Bild können z. T. noch lokale Umstände zur Erklärung herangezogen werden. $\mathrm{Da}$ jedoch die schnelle Picea-Ausbreitung noch in die limnische Phase fällt, inspiriert diese die Annahme einer spezifischen Wald- und damit auch der Klimaentwicklung, wie im folgenden noch auszuführen sein wird.

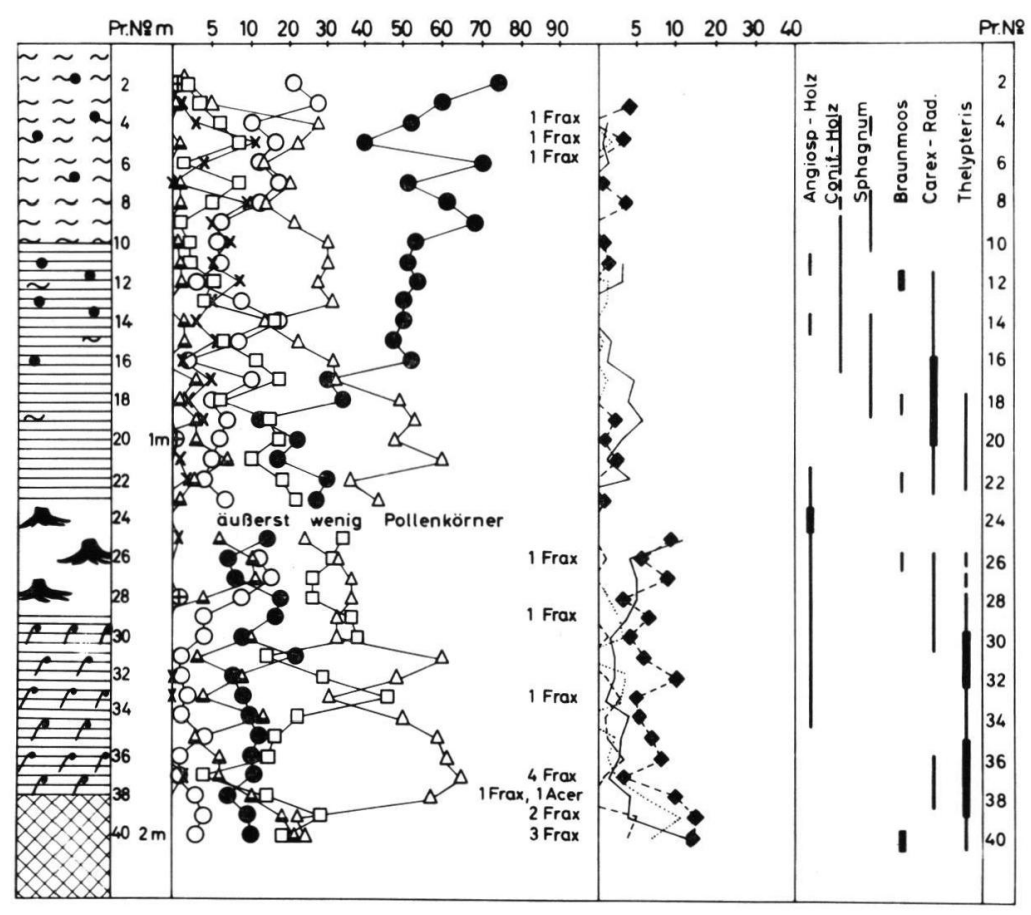

Abb. 3. Pollendiagramm der im Sommer 1959 aufgeschlossenen Folge. 
Aus bereits genannten Gründen liegen in der telmatischen Phase (Abb. 3) verschiedentlich bedingte Verzerrungen des Diagrammbildes vor. $\mathrm{Zu}$ diesen ist unbedingt der starke Picea-Anstieg am limno-telmatischen Kontakt zu rechnen. Andererseits besteht kein zwingender Grund zur Annahme einer selektiven Zerstörung. Eine gewisse Unsicherheit in der Beurteilung ist trotzdem nicht auszuschließen.

Die lokale Überrepräsentation von Alnus und Betula im Bereich des übrigens besonders im oberen Teil extrem holzreichen Bruchwaldtorfes ist verständlich. Diese verursacht im Diagrammbild die relative Unterdrückung der übrigen Elemente. Als lokale Überrepräsentation ist ebenso der hohe Anteil des Pinus- und Picea-Pollens im oberen Diagrammteil anzusehen. Im vorliegenden Falle kann nicht vom „Pinus-Anstieg am Ende der Eem-Warmzeit“" gesprochen werden. Dieser würde höchstens in den obersten $10 \mathrm{~cm}$ zu suchen sein.

Unter diesen Umständen gewinnt der relativ hohe prozentuale Anteil von Abies eine besondere Bedeutung. Trotz lokaler Unterdrückung im Diagramm durch Pinus und Picea erreicht Abies immer noch 7\% über mehrere Spektren. Die maximalen Werte liegen bei 9\% und $11 \%$. Ebenso bemerkenswert ist die relativ lang andauernde Anwesenheit. Ist doch Abies durch ca. $1 \mathrm{~m}$ stark gepreßten z. T. Carex-Torf, z. T. Sphagnum-Torf geschlossen vertreten. Sporadische Vorkommen beginnen bereits am limno-telmatischen Kontakt. Die europäischen Abies-Arten sind ausgesprochen subatlantisch-montane Elemente. Abgesehen von den Ursachen, die zur Ausbreitung von Abies im Waldbild der pleistozänen Warmzeiten im Flachlande führten, kann u. U. doch auf eine subatlantische Klimatönung in diesem Falle geschlossen werden, zumal auch die Moorbildung gleichzeitig eine subatlantische Klimatönung andeutet.

Der Typus der Moor- und auch der Waldentwicklung zeigt gewisse, wenn auch nicht offensichtliche Abweichungen von der gut bekannten Entwicklung während der EemWarmzeit. Während des Klima-Optimums bestanden noch keine wesentlichen Unterschiede zur Eem-Zeit. In der anschließenden Phase häufen sich - im Gegensatz zur EemZeit - die Anzeichen für ein subatlantisches Klima. Alle diese Abweichungen mit lokalen Faktoren erklären zu wollen, dürfte einige Schwierigkeiten bereiten. Zudem zeigt ein Pollenspektrum aus den stark gestörten humosen Einlagerungen in der Sandgrube von Osterwanna große Ähnlichkeit mit den hier behandelten Verhältnissen: Sphagnum-Torf von subatlantischer Tönung, Pinus 33\%, Betula 25\%, Alnus 15\%, Picea 17\%, Carpinus $3 \%$, Abies $7 \%$, Corylus 3\%.

Die hier geschilderte Vegetations- und Moor-Entwicklung liefert einige Argumente für die Annahme einer bisher nicht unterschiedenen pleistozänen Warmzeit. Die Schwierigkeiten der Trennung eines Eem-Bildes von dem einer problematischen Warmzeit vom Typ Bederkesa wären ja durch die geringen - obwohl auf einen unterschiedlichen Klimaablauf hinweisenden - Einzelheiten begründet und durchaus verständlich.

Ein weiteres Argument spricht für die tatsächlich unterschiedliche Entwicklung des hier beschriebenen Vorkommens gegenüber einem „normalen“ Eem-Bild: BendA \& SCHNEEKLOTH (1965) haben eine Bearbeitung des Interglazials von Köhlen veröffentlicht. Dieses befindet sich nur $6 \mathrm{~km}$ OSO von Bederkesa entfernt. Ein Vergleich beider benachbart gelegenen Vorkommen hinsichtlich der Vegetations-Entwicklung zeigt die Abweichungen in auffälliger Weise (wobei einige Punkte der Interpretation allerdings noch zu diskutieren wären). Jedenfalls entspricht aber das Pollendiagramm von Köhlen dem „normalen“ Eem-Bild. Schließlich sei noch erwähnt, daß Menke (1967) ebenfalls die Abweichungen zumindest für problematisch hält.

Trotzdem könnte die Belastung der Quartär-Stratigraphie mit einer weiteren problematischen Warmzeit des Saale-Komplexes, die in diesem Fall zwar biostratigraphisch fundiert scheint, für die aber bisher keine lithostratigraphische Bestätigung vorgelegt 
werden kann, als übereilt angesehen werden. Mehrere von verschiedenen Autoren publizierte Versuche haben sich bereits als solche erwiesen. Eine intensive Uberprüfung des bisher biostratigraphisch als eemzeitlich angesehenen Materials scheint in jeder Hinsicht lohnend zu sein.

\section{Literatur}

Benda, L. \& SchneeкLoth, H.: Das Eem-Interglazial von Köhlen, Krs. Wesermünde. Geol. Jb. 83, 699-716, 1965.

MenKe, B.: Ein Beitrag zur eemzeitlichen Vegetations- und Klimageschichte nach dem Profil von Ostrohe/Schleswig-Holstein. Fundamenta, Reihe B, 2, 126-135, 1967.

Manuskr. eingeg. 16. 9. 1965.

Anschrifț des Verf.: Dr. R. Hallik, Geologisches Landesamt Hamburg, 2000 Hamburg 13, Oberstraße 88 . 